\title{
Incidence of Patterns of Bony modification of Neck of the Talus in Indian Population
}

\section{Sandhya Nagolu ${ }^{1}$, Varalakshmi KL ${ }^{2}$, Sangeeta $\mathbf{M}^{3}$, Khizer Hussain Afroze M *4. \\ ${ }^{1}$ Lecturer, Department of Anatomy, MVJ Medical College \&Research Hospital, Hoskote, Banga-} lore, Karnataka, India.

2 Professor, Department of Anatomy, MVJ Medical College \&Research Hospital, Hoskote, Bangalore, Karnataka, India.

${ }^{3}$ Professor and Head, Department of Anatomy, MVJ Medical College \&Research Hospital, Hoskote, Bangalore, Karnataka, India.

${ }^{* 4}$ Assistant Professor, Department of Anatomy, MVJ Medical College \& Research Hospital, Hoskote, Bangalore, Karnataka, India.

\section{ABSTRACT}

Objective: The present study is an attempt to determine the various types of bony modifications on the dorsum of the neck of the human tali of both feet due to the continuous habit of squatting in humans and correlating these findings to the existing literature.

Materials and Methods: 70 tali of unknown sex, obtained from the Department of Anatomy of MVJ Medical College and Research Hospital, Bangalore were used for the study. The dorsal surface of talar neck was carefully examined for the presence of medial and lateral squatting facets, combined facets, continuous gutter shaped facets and extension of trochlear surface. The collected data were tabulated and percentage of each facets and trochlear extensions were calculated. Statistical analysis of data was performed.

Results: Out of 70 dry human tali, lateral squatting facets were present in 29 (41.4\%) bones and medial squatting facets were seen in 11 (15.7\%) bones, gutter shaped facets in $12(17.1 \%)$ tali and combined facet present in 10 (14.2\%) tali. Complete absence of facet was observed in 8 tali. Lateral extension of trochlear surface on the dorsum of neck was seen in 60 (85.7\%) bones and medial trochlear extension was seen in $8(11.5 \%)$ bones. Lateral squatting facets were more common on right side but medial, gutter and combined facets though less frequent were seen more on left side. Lateral extensions were more commonly seen than medial extensions.

Conclusion: The knowledge of incidence of these modifications on the dorsal surfaces of neck of the talus acts as a key anthropological factor to identify the racial and regional origin of unclaimed skeleton. Hence the results of this study will be of great help for forensic experts, anthropologist who are handling the unidentified skeletons.

KEY WORDS: Squatting Facets, Talus, Trochlear extensions, Sub Talar Joint Stability.

Corresponding Author: Dr. Khizer Hussain Afroze M, Assistant Professor, Department of Anatomy, MVJ Medical College \& Research Hospital, Hoskote, Bangalore, India. 562114.

E-Mail: drafroze.homoeo@gmail.com

Access this Article online

Quick Response code

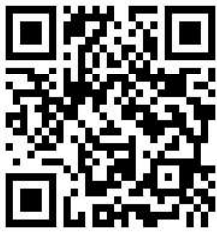

DOI: $10.16965 /$ ijar.2021.159

Journal Information

International Journal of Anatomy and Research ISSN (E) 2321-4287 | ISSN (P) 2321-8967

https://www.ijmhr.org/ijar.htm

DOI-Prefix: https://dx.doi.org/10.16965/ijar

Article Information

Received: 07 Aug 2021

Peer Review: 08 Aug 2021

Revised: 15 Sep 2021
Accepted: 21 Sep 2021

Published (O): 05 Dec 2021

Published (P): 05 Dec 2021

\section{INTRODUCTION}

Talus is one of the important tarsal bones of the human foot, it forms a link between the foot and the two long bones of the leg through the ankle joint [1]. The human foot plays a major role in locomotion and weight-bearing 
because of erect posture. As a reflection of individual behaviour and lifestyle, various bony modifications have been observed in skeletons of different populations. The position of the human body like erect posture, bipedal gait, and squatting position will put several stresses on the human foot leading to morphological variations [2].

Squatting position, most commonly practised in Indian races involves different posture at the hip, knee, and ankle joints i.e., hyperflexion at the hip and knee and hyper dorsiflexion at the ankle and subtalar joints [3]. Such habitual posture of squatting in humans leads to modifications of the ankle in the form of squatting facets in the talar neck and the trochlear surface of the talus as medial and lateral trochlear extensions. Squatting facets are evidence of specific forces to which the talus is exposed [4].

The anatomy of the skeleton of the bones is influenced by strenuous practices such as hunting and collecting, farming, scraping, and kneeling [5]. Results of previous studies have suggested, that the occurrence of squatting facets mainly depends on the different lifestyle activities, and in this modern era, since the lifestyle as well as squatting habits have been modified, there ma be a drastic change in the formation of squatting facets when compared to the earlier studies [6].

Squatting facets appears as an articular or smooth area on the dorsal surface of the talar neck which is not in line with the curvature of the trochlear surface. In some cases, squatting facets present as a transverse rim of bone without articular cartilage, separating the facets from the trochlear surface. In cases where there is a continuation between squatting facets and the trochlear surface, the concave shape of the facet changes instantaneously to continuous with the convex trochlear surface [4]. The medial aspect of the dorsal surface of the neck of the talus shows medial squatting facets which do not articulate with nor come in contact with the tibia whereas the lateral side of the neck of the talus presents smooth cartilage covered lateral squatting facets which come in contact with tibia during full dorsiflexion [3]. Trochlear extensions are defined as extensions of the trochlear surface which lie anterior to a line passing across the head of the talus perpendicular to the long axis of the foot, from the anterosuperior margin of the lateral malleolar surface to the medial malleolar surface [6].

The present study is an attempt to determine the various type of modifications on the dorsum of the neck of the human tali, variations in both feet and correlating these findings to the existing literature. Morphological knowledge of such variations is important in forensic anthropological studies as it would help in the identification of newly discovered skeleton remains of unknown cultural origin. Considering the population-based variations in lifestyle, this study would gather valuable data in the Indian population for designing ankle reconstruction surgeries [7].

\section{MATERIALS AND METHODS}

The current study was conducted on 70 (34 right and 36 left) dry human ossified tali of unknown sex obtained from the Department of Anatomy, MVJ Medical College and Research Hospital, Bangalore. Human tali free from any physical and pathological changes or deformities were included in the study. After getting approval from the institutional ethics committee, the dorsum of the neck of the individual talus was carefully examined for the appearance of medial and lateral squatting facets, combined facets, continuous gutter-shaped facets\& extensions of trochlear surface. The collected data were tabulated and the percentage of each facet and trochlear extensions were calculated.

\section{RESULTS}

The current study showed the presence of surface modification of the talus on the dorsum of the neck and trochlear surface in the form of squatting facets and trochlear extensions. [Figure 1 \& 2] In the present study, we observed four different types of squatting facets: Medial, Lateral, Combined and Gutter shaped. Out of 70 dry tali, 34 belonged to the right side and 36 were left-sided.

Out of 34 right human tali, lateral squatting facets were present in 17 (50\%) bones and medial squatting facets was seen in 5 (14.7\%) 


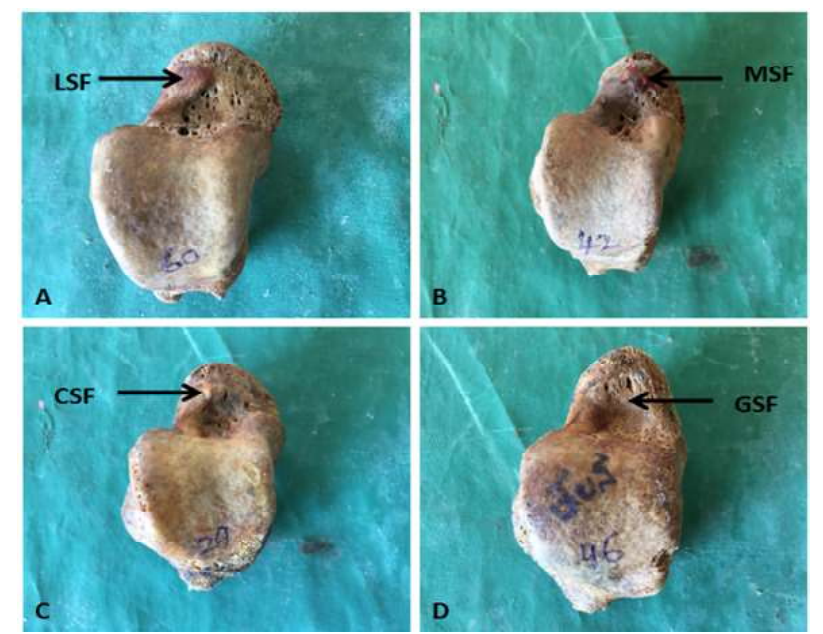

A: LSF - Lateral squatting facet

B: MSF - Medial squatting facet

C: CSF - Combined squatting facet

D: GSF - Gutter shaped facet

Fig. 1: Showing the different shapes of the facets.

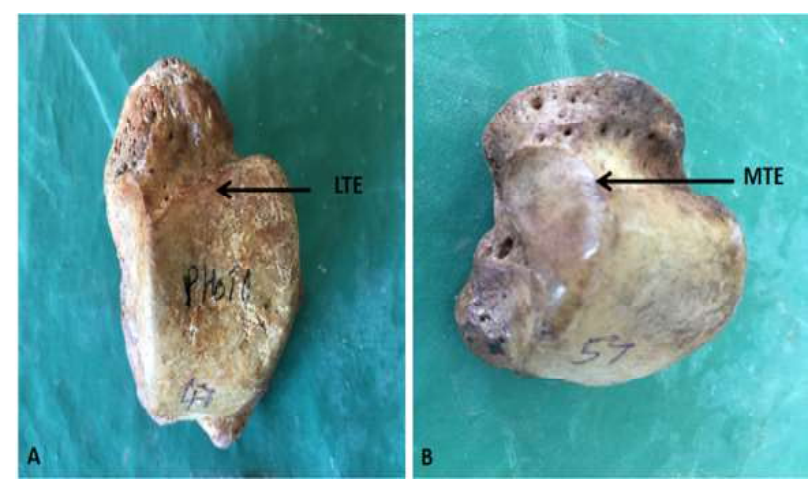

A: LTE - Lateral trochlear extension

B: MTE - Medial trochlear extension

Fig. 2: Showing the Trochlear extensions.

bones, gutter shaped facets in $5(14.7 \%)$ tali and combined facet was the least type observed i.e. in $4(11.8 \%)$ tali. Complete absence of facet was observed in 3 tali. The lateral trochlear extension was seen in 28 $(82.4 \%)$ bones and medial trochlear extension was seen in 6 (17.6\%) bones.

On the left side, out of 36 dry tali, 12 (33.3\%) bones showed lateral squatting facet, 6 (16.7\%) bones each showed medial and combined facet, Gutter shaped facet was observed in $7(19.4 \%)$ bones. In 5 bones there was a complete absence of facets. The trochlear surface on the dorsum of the neck showed medial trochlear extension in 2 (5.6\%) bones and lateral trochlear extension was seen in 32 (88.9\%) tali. [Table 1]

\section{DISCUSSION}

Continuous postural stress on the bones leads to change in the morphology of articular surfaces of human bones. Continuous habit of squatting as noticed in majority of Indians during the routine day to day chores such as washing, cleaning, pottery making, working in fields etc will lead to alteration in the structure of human skeleton especially of lower limb. To add on to this, Indian style toilets mainly in rural areas use squatting posture for defecating which is seldom observed in western population. One such modification as a result of this is the presence of squatting facets on the dorsal surface of neck of the talus and medial and lateral trochlear extensions [8]. Numerous studies have been conducted in the past in different races but very few studies of squatting facets are available in Indian literature. First detailed study was conducted by Thomson (1889) to detect the incidence of squatting facets on dorsal surface of talar neck and anterior margin of the distal extremity of tibia on 11 tali of Australian origin and 24 tali of Andamanese population. Their study revealed only lateral squatting facets in $63.64 \%$ of Australians and $50 \%$ of Andamanese [9].

In 1894 Charles and Wood JF conducted similar study on dry tali of Oriental races which also included foetuses and observed lateral squatting facets in $63 \%$ of talus. Their study provides evidence for the genetic inheritance of acquired characters $[10,11]$.

Table 1: Showing surface modification of right and left dry tali (squatting facets and trochlear extensions).

\begin{tabular}{lcccccc}
\hline \multirow{2}{*}{ Parameters } & \multicolumn{2}{c}{ Right (34 bones) } & \multicolumn{2}{c}{ Left (36 bones) } & \multirow{2}{*}{ Total (\%) } \\
\cline { 2 - 5 } & Number & Percentage & Number & Percentage & \\
\hline Medial squatting facet & 5 & 14.7 & 6 & 16.7 & 15.7 \\
Lateral squatting facet & 17 & 50 & 12 & 33.3 & 41.4 \\
Combined facet & 4 & 11.8 & 6 & 16.7 & 14.2 \\
Gutter shaped facet & 5 & 14.7 & 7 & 19.4 & 17.1 \\
Lateral trochlear extension & 28 & 82.4 & 32 & 88.9 & 85.7 \\
Medial trochlear extension & 6 & 17.6 & 2 & 5.6 & 11.4 \\
\hline
\end{tabular}


Barnett studied surface modification of talus in European population. According to Barnett, presence of squatting facets on the lateral aspect of talus is the distinct feature of Europeans (Seen in 2\% of individuals) which separates them from other races [12]. Study of 175 dry tali of Byzanthine origin by Oygucu et al showed the presence of lateral squatting facets in $37.7 \%$, medial and gutter shaped squatting facets in $0.6 \%$ [3].

Few Indian studies have been conducted on Morphology of squatting facets. Inderbir Singh studied 300 adults and 66 fetal tali using the Barnett's study as reference and concluded that the incidence of squatting facets is more in Indian adults than in the 35 Europeans. He observed lateral squatting facets in $28.6 \%$ whereas medial was absent. But while comparing it with foetus, he observed that the squatting facets were observed more in European foetus than Indian. Hence the author concluded that these facets in adults are mainly acquired due to habitual squatting pattern of Indians. IB Singh also suggested that the presence of such facets in fetus was mainly due to the considerable dorsiflexion of the foot during the intrauterine life [13].

Javia $M$ et al studied 221 dry tali and concluded that the occurrence of lateral squatting (51.5\%) facet is more in Indians in comparison to European, Egyptian and Byzanthine population. Medial squatting facet was seen in only $2.72 \%$ of population which was less compared to the other countries. In their study they also observed combined facets in $15.39 \%$ and absence of facets in $30.31 \%$ [2].

Study by Singh $\mathrm{N}$ et al on 616 tali showed the presence of lateral squatting facets in $50.81 \%$ and medial squatting facet in $1.79 \%$ [8]. Das conducted study on 200 dry tali of North Indian population and observed lateral squatting facet in $41 \%$, medial in $4 \%$ and combined facets in $3 \%$ of dry tali [14]. Pandey SK and Singh S conducted study on 262 dry tali showed $83.2 \%$ and $17.6 \%$ lateral and medial squatting facet respectively, $11.8 \%$ combined and $10.7 \%$ gutter shaped facet [15].

In the present study lateral squatting facet was seen in $41.4 \%$ which is in correlation with that Int J Anat Res 2021, 9(4):8104-08. ISSN 2321-4287 of study by Das and Oygucu. The incidence of Medial squatting facet, combined and gutter shaped facet was higher in the current study when compared to other studies.

The results of all the studies suggest that the frequency of appearance of squatting facets are variable depending on different races and Indian population shows higher incidence of these facets because of their life style habits. The previous studies have also suggested that increased incidence of lateral squatting facets are mainly due to the fact that during different postures body weight is distributed unequally more towards the lateral side of foot [4].

In the current study medial trochlear extension was seen in $11.4 \%$ of bone and lateral trochlear extension in $85.7 \%$ bones which is in correlation with the study by Thomson and Charles. Study by Barnett showed medial extension and lateral extensions of trochlear surface in $11 \%$ (similar to our study) and $17 \%$ of tali respectively and Barnett was the first one to prove that these extensions are different from squatting facets as he noted these extensions were not just confined to talar neck they continued on the anteroposterior curve of trochlea and during dorsiflexion they come in contact with inferior surface of tibia. So, he concluded that they are produced due to dorsiflexion of ankle joint rather than squatting position [12].

A study by Khadija lqbal et al on 300 dry tali showed presence of medial extension of trochlear surface in $34 \%$ tali and lateral extension in $58 \%$ bones. They concluded that the presence of such extensions is mainly due to the alteration in the orientation and shape of talar facets [16]. Oygucu observed medial trochlear extension in $10.9 \%$ and lateral trochlear extension in $8 \%$ of dry tali. [3] Medial trochlear extension was seen in $60.3 \%$ and lateral trochlear extension was seen 90.8 $\%$ of dry tali in a study conducted Pandey SK and Singh S [15].

Singh $\mathrm{N}$ et al observed medial trochlear extension in $43.18 \%$ and lateral trochlear extensions in $70.78 \%$ and suggested that high incidence of trochlear extensions in Indian population is may be attributed by squatting 
position, habitual sartorial posture or walking for a long distance [8].

\section{CONCLUSION}

Morphological variations of squatting facets in different races helps to identify the various habitual or daily activities that an individual in defined populations is involved in. The incidence of different types of squatting facets are due to either squatting position, cultural habits or genetic inheritance. The knowledge of incidence of these modifications on the dorsalsurfaces of neck of the talus acts as a key anthropological factor to identify the racial and regional origin of unclaimed skeleton. Hence the results of this study will add value for forensic experts, anthropologists who are handling the unidentified skeletons. Current study result also provides evidence that Indian population use squatting as a regular habit and the modifications of talus provide intensity and the regularity of the posture. Talar modifications are common in various populations, so it is advised to include it in standard textbooks of anatomy and osteology to assist teaching. Further studies on other populations groups are indicated using larger sample size to make comparative studies more meaningful.

\section{Conflicts of Interests: None}

\section{REFERENCES}

[1]. Susan Standring. Gray's Anatomy, The Anatomical Basis of Clinical Practice. 40th ed. Elsevier Churchill Livingstone Publications; 2008: Chapter 84.

[2]. Javia M, Changani M, Chudasama J, Thummar B, Vadgama J, Bambhaniya A. Morphological study of squatting facets on the neck of the talus in Indian population. J Res Med Den Sci 2014; 2(4):38-41.

[3]. Oygucu IH, Kurt MA, Ikiz I, Erem T, Davies DC. Squa4ing facets on the neck of the talus and extensions of the trochlear surface of the talus in Late Byzantine males. J Anat 1998; 192: 287-91.
[4]. Garg R, Shekhawat S, Mogra K, Kumar S. Modifi cations on Dorsum of Neck of Talus (Squatting Facets And Trochlear Extensions) in Indians. Acta Medica International. 2015; 2(1):100-104.

[5]. Kimberly Jones. Interpreting Behaviour from the Human Skeleton: Looking at Squatting Facets. Illinois University; 2007.

[6]. Shishirkumar, Nambiar S, Kumar A. Study of Squatting Facets in Tibia and Talus in South Indian Population. IJSR. 2014; 3(6): 2733 - 35.

[7]. Singh IB. Squatting facets on the talus and tibia in Indians. Journal of anatomy. 1959; 93: 540-50.

[8]. Singh N, Singh AK, Kumar R, Tiwari K, Chauhan NS. Re-Modelling of North Indian Talus: A Pressure Effect of Squatting Position. Ann. Int. Med. Den. Res. 2018; 4(3): 1-6.

[9]. Thomson A. The influence of posture on the form of the articular surfaces of the tibia and astragalus in the different races of man and the higher apes. J AnatPhysiol. 1889; 23: 616-39.

[10]. Charles RH. Morphological peculiarities in the Punjabi and their bearing on the question of the transmission of acquired characters. J AnatPhysiol. 1894; 28: 271-80.

[11]. Jones FW. Structure and Function as Seen in the Foot. Bailliere, Tindall and cox; 1944.

[12]. BARNETT CH. Squatting facets on the European talus. Journal of Anatomy. 1954; 88: 509-13.

[13]. Singh I. Squatting facets on the talus and tibia in Indian foetuses. ActaAnatomica. 1963; 54: 137-44.

[14]. DAS AC. Squatting facets of the talus in U.P. subjects. J Anat Soc India. 1959; 8: 90-92.

[15]. Pandey SK, Singh S. Study of squatting facet/ extension of talus in both sexes. Medicine, Science and the Law. 1990; 30(2): 159-64.

[16]. Khadija I, Ambreen S, Nadeem S. Anatomical variations of trochlear surface of talus. Journal of University Medical and Dental College 2012; 3(1): 38-41.

How to cite this article: Sandhya Nagolu, Varalakshmi KL, Sangeeta M, Khizer Hussain Afroze M Incidence of Patterns of Bony modification of Neck of the Talus in Indian Population. Int J Anat Res 2021;9(4):8104-8108. DOI: 10.16965/ ijar.2021.159 